\title{
PERCEPÇÃO DA ENFERMAGEM SOBRE A HIGIENE ORAL AO PACIENTE HOSPITALIZADO
}

\author{
Rodolfo de Oliveira Medeiros' ${ }^{1}$, Márcia Renata Rodrigues ${ }^{1}$, Márcia Aparecida Padovan Otani ${ }^{1}$ \\ Elza de Fátima Ribeiro Higa ${ }^{1}$ \\ 1Faculdade de Medicina de Marília, Brasil.rodorfo.famema@hotmail.com; marcia.renatarodrigues@gmail.com \\ mpadovanotani@gmail.com; hirifael@gmail.com
}

\begin{abstract}
Resumo. Objetivo: analisar a percepção, a importância e os fatores que interferem na higiene oral adequada ao paciente hospitalizado, na visão de enfermeiros, técnicos e auxiliares de enfermagem. Método: Pesquisa de campo qualitativa, realizada em um hospital do centro-oeste paulista com 16 participantes. A coleta de dados foi por meio de entrevista semiestruturada com duas perguntas direcionadoras sobre a percepção, importância e fatores que interferem na realização desse cuidado. Os dados foram analisados por meio da Técnica de Análise de Conteúdo na modalidade temática. Resultados: da análise emergiram duas categorias temáticas: 1. Relevância da higiene oral - apontam a organização e rotina e os materiais utilizados nesse cuidado e 2. Fatores que interferem na realização da higiene oral - alegam prioritariamente, falta de material e de recursos humanos para realização da higiene oral. Conclusões: os profissionais reconhecem a importância da higiene oral para manutenção da saúde e apontam a precarização do trabalho da enfermagem.
\end{abstract}

Palavras-chave: Higiene Bucal; Integralidade em Saúde; Equipe de Enfermagem.

\section{PERCEPTION OF NURSING ABOUT ORAL HYGIENE TO HOSPITALIZED PATIENTS}

\begin{abstract}
Objective: to analyze the perception, importance and factors that interfere with adequate oral hygiene for hospitalized patients, in the view of nurses, technicians and nursing assistants. Method: Field and qualitative research, carried out in a hospital in the Midwest of São Paulo with 16 participants. Data collection was carried out through a semi-structured interview with two guiding questions about the perception, importance and factors that interfere with this care. The data were analyzed using the Content Analysis Technique in the thematic modality. Results: two thematic categories emerged from the analysis: 1. Relevance of oral hygiene - points out the organization and routine and the materials used in this care and 2 . Factors that interfere in the performance of oral hygiene - they claim, primarily, lack of material and human resources to oral hygiene. Conclusions: professionals recognize the importance of oral hygiene for health maintenance and point out the precariousness of nursing work.
\end{abstract}

Keywords: Oral hygiene; Comprehensiveness in Health; Nursing Team.

\section{INTRODUÇÃO}

A higiene oral é um importante cuidado para a manutenção do conforto e integridade da mucosa da cavidade oral, além do controle de doenças bucais (MacNeill \& Sorenson, 2009). Pessoas com saúde bucal fragilizada podem sofrer impactos em seu estado de saúde geral, além de aspectos psicossociais como baixa autoestima e aceitabilidade social (Jerreat, Youssouf, Barker, \& Jagger, 2007).

Além de sua relevância no indivíduo saudável, práticas de higiene oral são de extrema importância no ambiente hospitalar. No indivíduo hospitalizado, cerca de 48 horas após sua 
admissão, sua flora oral é substituída por alguns microrganismos com alto potencial de virulência (Estaji, Alinejad, Hassan, \& Hackshani, 2015). A higiene oral inadequada pode resultar na formação de placas bacterianas, inflamação, dor e infecção (MacNeill \& Sorenson, 2009). Diante deste panorama, por manter contato direto com os pacientes, a equipe de enfermagem tem importante papel na saúde bucal do indivíduo hospitalizado, seja de forma educativa ou na execução de técnicas de higiene oral. Ressalta-se que a maioria das pesquisas relacionadas à temática higiene oral são realizadas em Unidades de Terapia Intensiva, sendo relevante estudos relacionados à higiene oral em unidades de cínica médica (Scalco, Fernandes, Scalco, Ribeiro, Shiguematsu, \& Poleti, 2019; Teixeira, Santos, \& Azambuja, 2019; Konuk, Aydin, Cangur, \& Ayen, 2019).

Ao se observar a rotina dos cuidados de enfermagem e, mais especificamente, às práticas de higiene oral, nos deparamos com um cuidado fragmentado (Chaves, Mininel, Silva, Alves, Silva, \& Camelo, 2017), evidenciando a pouca relevância dada pela equipe de enfermagem à esse aspecto como parte do cuidado integral. Diante do exposto e da compreensão do cuidado na perspectiva da integralidade, a relevância deste estudo se pauta na necessidade de compreender essa temática a partir da percepção dos profissionais de enfermagem, contribuindo, assim, para maior discussão e reflexão sobre a prática de enfermagem nos serviços hospitalares, além de estimular a busca de soluções para os fatores que dificultam esse cuidado. A pergunta que norteou essa pesquisa foi: Qual a importância da higiene oral como parte do cuidado integral ao indivíduo hospitalizado? Constituiu-se, portanto, como objetivo: Compreender a percepção, a importância e os fatores que interferem na higiene oral adequada ao paciente hospitalizado, na visão de enfermeiros, técnicos e auxiliares de enfermagem.

\section{MÉTODO}

Trata-se de uma pesquisa de campo qualitativa. Para Minayo a pesquisa qualitativa permite que o pesquisador alcance elementos relevantes de uma determinada realidade: desejos, aspirações, valores, dentre outros, tendo em vista a compreensão do objeto investigado. Nesse sentido, busca nitidez no ordenamento, coordenação e análise dos dados de processos ou fenômenos orientados por uma teoria. Suas principais características são: eficiência para indicar demandas reais; compreensíveis e relacionar-se contemplativamente entre fenômenos, fatos e coisas da realidade. O preparo da teoria tem como finalidade conseguir a compreensão do grupo de pensadores em uma determinada direção (Minayo, 
2016). A pesquisa foi realizada em três enfermarias de um hospital escola de uma cidade do oeste paulista, localizada a $450 \mathrm{~km}$ da capital. Essas enfermarias somam um total de 62 leitos operacionais. Para esta pesquisa, foram considerados participantes os profissionais de enfermagem, ou seja, Enfermeiros, Técnicos e Auxiliares de Enfermagem, que atuavam nessas três enfermarias. A população do estudo foi composta por 16 profissionais de enfermagem, das diferentes categorias, assim distribuídos: sete auxiliares de enfermagem, seis técnicos e três enfermeiros. As entrevistas tiveram duração média de 10 minutos. Foram excluídos os profissionais que trabalhavam há menos de 6 meses nas enfermarias. $A$ coleta de dados foi realizada no local de trabalho dos participantes, após contato prévio com os mesmos, por meio de entrevistas semiestruturadas. A entrevista semiestruturada é a técnica mais utilizada no processo de trabalho de campo (Minayo, 2013). O roteiro elaborado para entrevista contém dados sócio-demográficos e perguntas abertas com a finalidade de investigar a percepção que possuem sobre a importância da higiene oral e como realizam essa prática no paciente hospitalizado. As entrevistas foram gravadas e transcritas pelo próprio pesquisador objetivando assegurar o sigilo acordado. O período da coleta de dados foi entre os meses de abril e maio de 2018. A coleta de dados foi interrompida por meio da estratégia de saturação teórica, que cumpre a função operacional, que indica o momento do pesquisador parar a coleta de dados evitando custos e prolongamentos de tempo da pesquisa (Sousa, Erdmann, \& Magalhães, 2016).

Dentre as várias modalidades de análise de conteúdo, optou-se pela técnica de Análise de Conteúdo na modalidade Temática, constituída pelas fases Pré análise, Exploração do material e Interpretação dos resultados (Bardin, 2012). Na primeira fase é realizada leitura exaustiva dos dados obtidos, com a finalidade de aprofundar o conteúdo, elaborar pressupostos para sustentação da análise e a interpretação do material. As ideias iniciais organizam um plano de análise, a partir de pressupostos, da leitura flutuante, tendo em vista os objetivos a serem alcançados, constituindo-se um corpus de análise (Bardin, 2012).Na fase de Exploração do material é realizada a categorização dos dados, recortando no texto as unidades de registro, que podem ser palavras, frases, expressões significativas, personagem, temas considerados relevantes para o pesquisador, a partir das unidades de registro e de contexto. Em seguida, a codificação das unidades de registro, a classificação e a organização dos dados, delimitando as categorias teóricas ou empíricas a serem utilizadas (Bardin, 2012). A exploração do material visa alcançar o núcleo de compreensão do texto. Os núcleos de núcleos de sentidos são alinhados aos objetivos da pesquisa. As categorias 
temáticas são estruturadas segundo os critérios: 1- exaustão, estas devem conter todo material; 2- Representatividade, a porção analisada necessita ser representativa da natureza da pesquisa: 3- exclusão mútua, cada tema não pode existir em mais de uma categoria; 4- homogeneidade, organiza-se, num mesmo conjunto categorial; 5- pertinência, dados pertencentes ao quadro teórico; 6- objetividade e 7-fidedignidade para que não sobreponha a subjetividade do pesquisador; 8- Concretude, não serem expressas de forma abstrata que podem sugerir muitos significados, e 9- produtividade, ao proporcionar resultados relevantes (Minayo, 2013, Bardin, 2012).

Na última fase, interpretativa, ocorre a análise aprofundada sobre os dados obtidos, que são relacionados aos significantes e aos significados das mensagens. A interpretação surge da articulação entre a fundamentação temática e teórica metodológica, das perguntas da pesquisa, dos resultados obtidos e do objetivo a ser alcançado (Minayo, 2013, Bardin, 2012).

As entrevistas foram realizadas mediante a Resolução ํo 466, de 12 de dezembro de 2012, que regulamenta a pesquisa envolvendo seres humanos, assegurando seus direitos e deveres, na qual deve ser submetida ao sistema do Conselho de Ética em Pesquisa (CEP). Após a autorização da Diretoria do Hospital onde foi realizado a pesquisa e a aprovação no Comitê de Ética em Pesquisa Envolvendo Seres Humanos, sob o número de parecer 2.517.992, e CAAE 79191317.5.0000.5413, a entrevista foi realizada mediante o fornecimento das informações referentes ao objetivo do estudo, a forma de participação e a livre opção da pessoa em participar ou não, sem qualquer prejuízo em seu trabalho e após leitura e assinatura do Termo de Consentimento Livre e Esclarecido. Desse modo, considerando os aspectos éticos os participantes foram assim identificados: letra $E$ (Entrevista) seguida de número (1 a 16), indicando a ordem em que as entrevistas foram realizadas.

\section{RESULTADOS}

\subsection{Dados dos participantes}

A faixa etária dos entrevistados variou entre, 33 e 54 anos. O tempo de profissão foi de 1 a 25 anos e 14 do sexo feminino e dois masculinos, esse último dado corrobora a literatura no qual aponta para a predominância feminina da equipe de enfermagem. 


\subsection{Análise dos dados}

Da análise dos dados obtidos emergiram duas categorias temáticas: 1. Relevância da higiene oral e 2. fatores que interferem para a realização da higiene.

\subsubsection{Relevância da higiene oral}

Ao abordarem a importância da higiene oral os participantes também acharam relevante falar toda sua rotina de trabalho, ao receberem Plantão:

Bom, a gente segue né, primeiro a rotina de controle, depois medicação, e depois começa os cuidados de higiene e conforto né, banho, higiene oral, troca (E1).

A rotina de trabalho do enfermeiro está relacionada a organização e distribuição de atividades da equipe de enfermagem, além de visita de enfermagem.

Eu chego, pego o plantão, depois eu vou organizar o plantão, vejo os funcionários, quem vai ficar na medicação, quem vai ficar nos cuidados, banhos, essas coisas, faço os pedidos de materiais necessários segundo uma escala que temos, depois eu vou priorizar o que eu tenho em relação a buscar paciente, e depois passo visita (E6).

Apontaram também a gravidade e idade dos pacientes e o grau de dependência como itens a serem priorizados na rotina de cuidado.

A gente costuma priorizar mesmo os idosos, por que por exemplo, a gente vai fazer uma higiene do couro cabeludo, geralmente eles não gostam de fazer muito tarde, por que em casa, tomam banho cedo. Então a gente quer ir neles primeiro (E4).

Quando conversado acerca da importância da higiene oral, todos concordam que tal prática é importante, mas admitem que não seja a prioridade.

Ah, [a higiene oral] dá uma sensação de bem estar, é uma maneira do paciente se sentir limpo também. Infelizmente não é uma coisa que é feita regularmente. Infelizmente o pessoal não dá muita importância (E2).

Outros profissionais relataram que tal prática é importante na prevenção de doenças, como endocardite e pneumonia.

A higiene oral é muito importante mesmo, até por conta de proliferação de bactérias na cavidade oral, até nessa questão de secreção, pneumonia, a sujidade nessa área aí pode acarretar(E9).

Em relação à técnica de higiene oral, percebe-se que os pacientes em ventilação mecânica possuem prioridade no momento dos cuidados de higiene oral. 
Bom, o paciente entubado é a gente que realiza mesmo né. O paciente parcialmente dependente, depende do grau de dependência do paciente né (E1).

$E$ É, na verdade eu vou ter que ser sincera, a orientação a gente não faz (E7).

Quanto aos materiais utilizados, obtivemos dois tipos, quando pertence ao paciente e quando é fornecido pelo hospital. A maioria dos participantes relataram usar antisséptico bucal e creme dental, além de usarem o aspirador em casos de pacientes em ventilação mecânica.

Nós usamos o enxaguante bucal, que a gente as vezes pede pra família tá trazendo, [...] que o paciente tiver costume de utilizar em casa, ou a pasta de dente. Nós utilizamos também a clorexedina aquosa diluída em um pouco de água (E9).

\subsubsection{Fatores que interferem na realização da higiene oral}

Para se compreender a percepção dos profissionais de enfermagem acerca dos fatores que interferem na realização do cuidado de higiene oral, foi necessário destacar os fatores que contribuem e os que prejudicam:

Quando tem material né, quando tem as coisas aí fica tudo mais fácil né, por que muitos não têm condição de comprar nada, nem uma escova, nem um antisséptico bucal, então o que auxilia é ter as coisas pra fazer a higiene oral né (E10).

Entre os fatores que prejudicam a execução da técnica, falta de funcionários é o relato mais evidenciado nas entrevistas.

Falta de funcionários, falta de mão de obra mesmo né, por que é muito mais fácil trabalhar com o número de funcionários completo né, que a assistência na verdade é muito melhor, não sobrecarrega o funcionário, o paciente sai ganhando, a gente consegue prestar uma assistência de qualidade(E1).

O número de funcionários né. Por que muitas vezes a gente trabalha com o número de funcionários reduzidos, aí a gente dá prioridade pro banho mesmo né, e certos cuidados acabam ficando pra trás e a desejar né (E2).

Outros profissionais relataram que a falta de supervisão interfere na não prestação do cuidado de higiene oral.

O que prejudica um pouco é a falta de fiscalização né (E8). 


\section{DISCUSSÃO}

No ambiente hospitalar, embora a prática de higiene oral pertença à equipe de enfermagem, é frequentemente negligenciada (Noort, Witteman, Hartog, \& Everaars, 2019), seja por falta de conhecimento acerca da importância do procedimento, ou por falta de cuidados de rotina que contemplem a higiene oral como procedimento padrão (Orlandini, 2012). Ficou evidente nas falas dos entrevistados, que a grande preocupação da equipe de enfermagem consiste em cumprir as escalas de trabalho, pautando-se ainda no modelo funcionalista, influenciados por Frederick W. Taylor (1856-1915), e Jules Henri Fayol (1841-1925), (Tsuji, \& Aguilar-da-Silva, 2010). A rotina de cuidados de auxiliares e técnicos de enfermagem consiste em: verificação de parâmetros vitais, preparo e administração de medicamentos, cuidados de higiene e conforto, controle de ingestão e eliminações e coleta de material para exames (Souza, Santos, \& Monteiro, 2013). Em relação à rotina dos enfermeiros, a mesma é dividida em atividades gerenciais, como passagem de plantão, controle da assiduidade dos funcionários e elaboração das escalas de serviços, atividades assistenciais, como visita de enfermagem, liderança e tomada de decisão (Lee, Noh, Han, \& Jeon, 2019). Porém, devido à alta demanda do serviço de saúde, enfermeiros dedicam a maior parte do tempo a atividades gerenciais.

Referente às prioridades de cuidado estabelecidas pela equipe de enfermagem, banho, cuidados de higiene e mudança de decúbito são as mais valorizadas entre auxiliares e técnicos de enfermagem (Passos, 2011). Nos depoimentos dos entrevistados, é nítida a percepção de que o profissional da enfermagem se sente incomodado em ver o paciente com higiene deficitária em decorrência de eliminações fisiológicas, seja por questões de higiene, seja pela aparência. Entre os enfermeiros, as prioridades devem estar voltadas para o gerenciamento, planejamento e avaliação dos cuidados prestados por parte de sua equipe (Soares, Leal, \& Resck, 2019). Partindo do pressuposto de que necessidades de saúde consiste em um conjunto de coisas indispensáveis à vida (Tsuji, \& Aguilar-da-Silva, 2010), cuidados como higiene e conforto são indispensáveis a vida do ser humano.

Portanto, a falta da higiene oral favorece o aparecimento e desenvolvimento de bactérias na cavidade oral, aumentam as chances de exposição a comorbidades, como pneumonia, além de causar comprometimento do estado imunológico (Lee, Noh, Han, \& Jeon, 2019). Desta forma, é possível compreender a importância da higiene oral como prática preventiva. No presente estudo, a disponibilização de materiais foi apontada como fator primordial para a 
realização da técnica de higiene oral. Uma adequada gestão de materiais influencia diretamente no desempenho da organização em geral, proporcionando segurança no cuidado (Bogo, Bernardino, Castilho, \& Cruz, 2015). A falta de funcionários, a falta de materiais adequados, o modelo de trabalho por divisão de tarefas, a limitada disponibilidade de produtos, a carga excessiva de trabalho de enfermagem atrelada a falta de tempo e a falta de uma rotina de cuidados relacionados a higiene oral baseado em evidências influenciam na não prestação de cuidados de enfermagem, em específico, higiene oral, além de outros importantes cuidados indispensáveis ao indivíduo hospitalizado, causando insatisfação do paciente (Noort, Witteman, Hartog, \& Everaars, 2019), (Recio-Saucedo, Dall'Ora, Maruotti, Ball, Briggs, Meredith, Redfern, Kovacs, Prytherch, Smith, \& Griffiths, 2018), (Coker, Ploeg, Kaasalainen, \& Carter, 2017), Gibney, Wright, \& Naganathan, 2019).

\section{LIMITAÇÕES DO ESTUDO}

As limitações do estudo incluem o fato das entrevistas terem sido realizadas no local e horário de trabalho, competindo com uma rotina já exaustiva e sobrecarregada e também a escassez de pesquisas sobre o tema com a mesma população alvo, o que limitou a comparação de resultados com outros estudos realizados

\section{CONSIDERAÇÕES FINAIS}

Na visão dos profissionais de enfermagem, a higiene oral é importante medida na promoção de conforto e bem-estar do paciente, ou na prevenção de doenças. Porém, apesar de ser considerada como um importante cuidado esta técnica não é priorizada entre os profissionais de enfermagem no momento da assistência ao paciente hospitalizado. É evidente que a falta de recursos materiais, atrelada ao número reduzido de funcionários, interferem de forma negativa na assistência ao paciente, além da sobrecarga de atividades e, consequentemente, desmotivação dos profissionais de enfermagem. O hospital possui um Procedimento Operacional (PO), porém, ele não é utilizado, além de estar desatualizado. Tal fato surge como um fator que prejudica a assistência da equipe de enfermagem, fazendo com que cada profissional realize a técnica de uma forma, sem um eixo a ser seguido. Além disso, a utilização de um PO serve como ferramenta para que a equipe possa se respaldar e se orientar. A partir desta pesquisa, sugere-se que o Procedimento Operacional Padrão (PO) de Higiene Oral da instituição seja atualizado, para que o mesmo venha ser utilizado e valorizado pelos funcionários da instituição. 
Em um sistema hierarquizado e não-universal, a consciência técnica dos profissionais envolvidos no cuidado ao indivíduo hospitalizado deve estar alinhada às necessidades de saúde dos usuários dos serviços de saúde. Para tanto, os resultados do presente estudo ilustram a iminente necessidade de conscientização por parte dos gestores e profissionais de enfermagem acerca dos cuidados prestados ao indivíduo hospitalizado, além de ações de reflexão acerca do atual modelo assistencial, pautado na divisão por tarefas, contrapondo-se à ótica de cuidado integral, ressaltando a indispensável ótica biopsicossocial como eixo estruturante do cuidado, atrelado a ações de conscientização, para que esta técnica seja vista não como algo opcional, mas como parte relevante do cuidado.

\section{REFERÊNCIAS}

Bardin L. (2012). Análise de conteúdo. São Paulo (SP): Almedina Brasil.

Bogo, P. C., Bernardino, E., Castilho, V., \& Cruz, E. D. A. (2015). The nurse in the management of materials in teaching hospitals. Revista da Escola de Enfermagem da USP, 49(4), 629-635.

Chaves, L. D. P., Mininel, V. A., Silva, J. A. M., Alves, L. R., Silva, M. F., \& Camelo, S. H. H. (2017). Supervisão de enfermagem para a integralidade do cuidado. Revista Brasileira de Enfermagem, 70(5), 1165-1170.

Coker, E., Ploeg, J., Kaasalainen, S., \& Carter, N. (2017). Nurse's oral hygiene care practices with hospitalized older adults in post a cute seetings. International Journal of Older People Nursing, 12, e12124.

De Marco, A. A., Cardoso, C. G., De MArco, F. V, C., Melo Filho, A. B., Santamaria, M. P., \& Jardini, M. A. M. (2013). Oral confdition of critical patientes and its correlation with ventilator associated pneumonia: a pilot study. Revista de odontologia da UNESP, 42(3), 182-187.

Estaji. Z., Alinejad, M., Hassan Hackshani, M., \& Rad, M. (2015). The comparison of Chlorhexedine solution and swab with toothbrush and toothpaste effect on preventing oral lesion in hospitalized patients in intensive care unit. Global journal of Health Science, 8(5), 211-216.

Gaetti-Jardim, E. G., Setti, J. S., Chaede, M. F. M., \& Mendonça, J. C. G. (2013). Atenção odontológica em pacientes hospitalizados: Revisão de literatura e proposta de protocolo de higiene oral, Revista Brasileira de Ciências da Saúde, 11(35), 31-36.

Gibney, J. M., Wright, F. A., \& Naganathan, V. (2019). Improving the oral health folder people in hospital. Australasian Journal on Agening, 38(1), 33-38.

Jerreat, M., Youssouf, N., Barker, C., \& Jagger, D. C. (2007). Denture care of in-patients: the views of nursing staff and the development of an educational programme on denture care. Journal of research in Nursing, 12(2), 193-199.

Konuk, D. S, Aydin, M., Cangur, S., \& Guyen, E. (2019). The effect of oral care with clorhexedine, vitamin E and honey on mucosits in pediatric intensive care patientes. Journal of Pediatric Nursing, 45.

Leal, L. A., Soares, M. I., Silva, B. R., Bernardes, A., \& Camelo, S. H. H. (2018). Competências clínicas e gerais para enfermeiros hospitalares: visão de egressos de enfermagem. Revista Brasileira de Enfermagem, 71(4), 1605-1612.

Lee, Y. J., Noh, J., Han, S. Y., \& Jeon, H. S. (2019). Oral health care provided by nurses for hospitalized patients in Korea. International Journal of Dental Hygiene, 17, 336-342. 
MacNeil, B. A., \& Sorenson, H. M. (2009). Oral hygiene for the ventiled patient. AARC Times Magazine, 15.

Minayo, M. C. S. (2013). O desafio do conhecimento: pesquisa qualitativa em saúde. São Paulo: Hucitec.

Minayo MCS. (2016). O desafio da pesquisa social. In: Minayo MCS, Deslandes SF, Gomes R. Pesquisa social: teoria, método e criatividade. Petrópolis (RJ): Vozes; p. 9-28.

Noort, H. V., Witteman, B. J. M., Hertog, R. D., \& Everaars, B. (2019). A context analysis on how oral care is delivered in hospitalized patientes: a mixed-methods study. Journal of Clinical Nursing, 1-13.

Orlandini, G. M. (2012). Conhecimento da equipe de enfermagem sobre higiene oral em pacientes criticamente enfermos. Revista Gaúcha de Enfermagem, 33(3), 34-41.

Passos, S. S. S. (2011). Cuidados de enfermagem ao paciente dependente e hospitalizado. Revista de Enfermagem UFRJ, 19(4), 598-603.

Recio-Saucedo, A., Dall'Ora, C., Maruotti, A., Ball, J., Briggs, J., Meredith, P., RedFern, O. C., Kovacs, C., Prytherch, D., Smith, G. B., \& Griffiths, P. (2018). What impact does nursing care left undone have on patient outcomes? Review of Literature. Journal of Clinical Nursing, 27(12), 2248-2259.

Scalco, J. M., Fernandes, T. M. F., Scalco, V. F., Ribeiro, E., Shiguematsu, S. M., \& Poleti, M. L. (2019). Analysys of preventative influene on a oral hygiene protocol on ventilator-associated pneumonia. Journal of Health Sciences, 21(3), 281-283.

Soares, M.I., Camelo, S. H. H., Resck, Z. M. R., \& Terra, F. S. (2016). Saberes gerenciais do enfermeiro no contexto hospitalar. Revista Brasileira de Enfermagem, 69(4), 676.

Soares, M. I., Leal, M. A., REsck, M. R. M, Terra, F. S., Chaves, L. D. P., \& Henriques, S. H. (2019). Competence-based performance evalution in hospital nurses. Revista Latino-Americana de Enfermagem, 27:e3184.

Souza, F. G. M., Erdmann, A. L., \& Magalhães, A. L. P. (2016). Contornos conceituais e estruturais da pesquisa qualitaitva. Porto Alegre: Moriá.

Souza, M. F. G., Santos, A. D. B., \& Monteiro, A. I. (2013). O processo de enfermagem na concepção de profissionais de enfermagem de um hospital de ensino. Revista Brasileira de Enfermagem, 66(2), 167-173.

Teixeira, K. C. F., Santos, L. M., \& Azambuja, F. G. (2019). Análise da eficácia da higiene oral de pacientes internados em unidades de terapia intensiva de um hospital de alta complexidade no sul do Brasil. Revista de Odontologia da Unesp, 31(2), 134-144.

Tsuji, H., \& Aguilar-da-Silva, A. (2010). Aprender e ensinar na escola vestida de branco: do modelo biomédico ao humanístico. São Paulo: Phorte. 